\title{
Effect of steriles states on lepton magnetic moments and neutrinoless double beta decay
}

\author{
A. Abada, ${ }^{a}$ V. De Romeri ${ }^{b}$ and A.M. Teixeira ${ }^{b}$ \\ ${ }^{a}$ Laboratoire de Physique Théorique, CNRS - UMR 8627, \\ Université de Paris-Sud 11, F-91405 Orsay Cedex, France \\ ${ }^{b}$ Laboratoire de Physique Corpusculaire, CNRS/IN2P3 - UMR 6533, \\ Campus des Cézeaux, 24 Av. des Landais, F-63177 Aubière Cedex, France \\ E-mail: asmaa.abada@th.u-psud.fr, deromeri@clermont.in2p3.fr, \\ ana.teixeira@clermont.in2p3.fr
}

\begin{abstract}
We address the impact of sterile fermion states on the anomalous magnetic moment of charged leptons, as well as their contribution to neutrinoless double beta decays. We illustrate our results in a minimal, effective extension of the Standard Model by one sterile fermion state, and in a well-motivated framework of neutrino mass generation, embedding the Inverse Seesaw into the Standard Model. The simple " $3+1$ " effective case succeeds in alleviating the tension related to the muon anomalous magnetic moment, albeit only at the $3 \sigma$ level, and for light sterile states (corresponding to a cosmologically disfavoured regime). Interestingly, our analysis shows that a future $0 \nu 2 \beta$ observation does not necessarily imply an inverted hierarchy for the active neutrinos in this simple extension. Although the Inverse Seesaw realisation here addressed could indeed ease the tension in $(g-2)_{\mu}$, bounds from lepton universality in kaon decays mostly preclude this from happening. However, these scenarios can also have a strong impact on the interpretation of a future $0 \nu 2 \beta$ signal regarding the hierarchy of the active neutrino mass spectrum.
\end{abstract}

Keywords: Rare Decays, Beyond Standard Model, Neutrino Physics

ARXIV EPRINT: 1406.6978 


\section{Contents}

1 Introduction 1

2 Sterile neutrinos and lepton magnetic moments 3

3 Sterile neutrino extensions of the SM 5

3.1 Constraints on sterile neutrino models 5

3.2 Effective SM extension: "3+1" model 8

3.3 The inverse seesaw scenario 9

4 Analysis and discussion $\quad 10$

4.1 Results: " $3+1$ ” effective model 11

$\begin{array}{ll}4.2 & \text { Results: inverse seesaw scenario } \\ \end{array}$

$\begin{array}{lll}5 & \text { Conclusions } & 19\end{array}$

\section{Introduction}

Extensions of the Standard Model (SM) by sterile neutrinos have received increasing attention in recent years: in addition to their rôle in numerous well motivated scenarios of neutrino mass generation, the existence of sterile neutrinos is suggested by the reactor [1$3]$, accelerator [4-7] and Gallium anomalies [8,9], as well as by certain indications from large scale structure formation $[10,11]$.

Depending on their mass (which can range from well below the electroweak to the Planck scale), and more importantly, on their mixing with the active neutrinos, the sterile fermions can lead to non-negligible modifications of the leptonic charged current interaction $(W \ell \nu)$, which would be manifest in electroweak (EW) interactions as a deviation from unitarity of the leptonic mixing matrix $[12,13]$; in turn this will have an impact on numerous observables, providing contributions to lepton flavour violating (LFV) processes [14-17], leading to the violation of lepton flavour universality (LFU) [18-21], and enhancing rare leptonic (tau) decays, leptonic and semi-leptonic meson decays [22], also impacting invisible $Z$-boson [23] and Higgs boson decays [24-28].

Sterile neutrinos could also have a relevant rôle in flavour conserving observables, as is the case of electric and magnetic lepton moments, neutrinoless double beta decay [29], and several lepton number violating (LNV) decays, as for example $B^{-} \rightarrow h^{+} \ell^{-} \ell^{-}(h$ denoting a meson) among others currently being explored by the LHC collaborations (see, for example, [30] and references therein). Although the anomalous magnetic moment of the electron, which is now experimentally determined to an impressive precision [31], exhibits a striking agreement with the SM theoretical prediction, the same does not occur for the 
muon's. In fact, the $3.6 \sigma$ discrepancy [32] between the SM prediction and the corresponding measurements, $\Delta\left(a_{\mu}\right)$, strongly suggests that some new physics might be required in order to reconcile theory and observation.

In this work we investigate whether the sterile fermions can provide new contributions to the muon anomalous magnetic moment, possibly alleviating the current tension. For this, we consider the SM minimally extended ${ }^{1}$ by new sterile fields (for example right-handed neutrinos and/or other pure fermionic singlet states). The most minimal of these extensions consists in an effective, ad-hoc model, where one sterile state is added to the neutral fermion content of the SM. In this so called " $3+1$ " effective framework, the additional state encodes the effect of a given (arbitrary) number of sterile fermions, and their mixings with the active (light) neutrinos. In this approach no assumption is made concerning the underlying mechanism of neutrino mass generation. On the other hand, sterile neutrinos are a crucial ingredient of many well-motivated mechanisms accounting for neutrino masses and mixings; their impact on many of the above mentioned observables is particularly important in scenarios where the sterile states are comparatively light - as is the case of the $\nu \mathrm{SM}[34]$, the low-scale type-I seesaw [35] and the Inverse Seesaw (ISS) [36], among other possibilities. The ISS, where both sterile and right-handed neutrinos are added to the SM field content, is particularly appealing as it can be realised at low scales for natural values of the neutrino Yukawa couplings. Here, and as an illustrative example of a low-scale model of neutrino mass generation we considered a realisation of the ISS mechanism with 3 right-handed neutrinos and 3 additional sterile states (from now on labeled for simplicity "ISS model").

Notice however that all these frameworks are severely constrained, from both a theoretical and an observational point of view, and any realisation must comply with an extensive array of bounds. In addition to accommodating neutrino data [37-41], these extensions must comply with unitarity bounds [42, 43], laboratory bounds [44], electroweak precision tests [23, 45, 46], LHC constraints (as those arising from Higgs decays) [24-28], bounds from rare decays $[14,15,21,22]$ as well as cosmological constraints $[10,47]$. New sources of lepton number violation can trigger neutrinoless double beta decay (see, for example, [29]), and the sterile states can contribute to the decay rate: the additional mixings and possible new Majorana phases might enhance the effective mass, potentially rendering it within experimental reach, or even leading to the exclusion of certain regimes due to conflict with the current bounds (the most recent results on neutrinoless double beta decay have been obtained by the GERDA experiment [48]).

Motivated by the intense experimental activity in searching for a first signal of neutrinoless double beta $(0 \nu 2 \beta)$ decay, and in parallel to the study of the lepton magnetic moments, we also explore in this work the impact of the sterile fermionic states regarding neutrinoless double beta decay. Our analysis reveals that scenarios with sterile fermions can indeed contribute to alleviate the $(g-2)_{\mu}$ tension and, more importantly, can have a strong impact on the interpretation of a future $0 \nu 2 \beta$ signal regarding the hierarchy of the active neutrino mass spectrum.

\footnotetext{
${ }^{1} \mathrm{~A}$ model-independent study of an explanation for the observed discrepancy in the muon anomalous magnetic moment and new physics searches at the LHC has recently been carried in [33].
} 
Our work is organised as follows: in the subsequent section, we address the contributions to the anomalous lepton magnetic moments in the presence of sterile neutrinos, also discussing their impact for neutrinoless double beta decay. Section 3 is devoted to sterile neutrino extensions of the SM: we discuss in detail the different constraints, and present the two minimal models which will be subsequently explored - the " $3+1$ " effective model, and the ISS. Our numerical results for both models are collected in section 4, where we conduct a comprehensive analysis of the corresponding parameter spaces, and discuss the results. We summarise the most relevant points in the Conclusions.

\section{Sterile neutrinos and lepton magnetic moments}

The magnetic moment of a charged lepton is given by

$$
\vec{M}=g_{\ell} \frac{e}{2 m_{\ell}} \vec{S}
$$

with $\vec{S}, e$ and $m_{\ell}$ the charged lepton's spin, electric charge and mass. Higher order (loop) effects lead to small calculable deviations from the (Dirac) value $g_{\ell}=2$, so that the anomalous magnetic moment is defined as

$$
a_{\ell}=\frac{g_{\ell}-2}{2} .
$$

The experimental measurement of the anomalous magnetic moment of the electron differs from its SM theoretical expectation by

$$
\Delta\left(a_{e}\right)=-10.5(8.1) \times 10^{-13},
$$

its theoretical prediction being dominated by QED contributions, which have been calculated up to five loops $[49,50]$. New physics contributions to $\Delta\left(a_{e}\right)$ are generally assumed to be very small (although in [51] it was suggested that this observable could be used to probe and constrain new physics scenarios, an idea also recently explored in [52]); in fact, the precision in the determination of $a_{e}$ has rendered it the preferred means to determine the value of the fine-structure constant $\alpha$ (see, for example, [53]).

On the other hand, the anomalous magnetic moment of the muon has revealed a (yet unresolved) discrepancy between the SM expected value and the experimental determination. The current experimental (averaged) result is given by [53]

$$
a_{\mu}^{\exp }=11659209.1(5.4)(3.3) \times 10^{-10} .
$$

The SM prediction for $a_{\mu}^{\mathrm{SM}}$ is generally divided into three contributions [54-59],

$$
a_{\mu}^{\mathrm{SM}}=a_{\mu}^{\mathrm{QED}}+a_{\mu}^{\mathrm{EW}}+a_{\mu}^{\mathrm{had}} .
$$

Hadronic (quark and gluon) loop contributions to $a_{\mu}^{\mathrm{SM}}$ are the ones most affected by theoretical uncertainties. By combining the different contributions, one has [32]

$$
a_{\mu}^{\mathrm{SM}}=116591803(1)(42)(26) \times 10^{-11},
$$




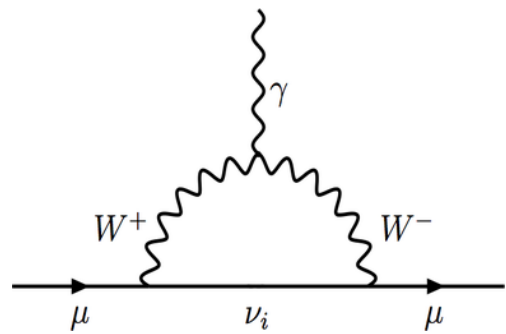

Figure 1. One loop Feynman diagram contributing to the anomalous magnetic moment of charged leptons, involving the weak gauge bosons $W^{ \pm}$and the neutral fermions $\nu_{i}$, including the extra sterile states.

where the errors are due to the electroweak, lowest-order hadronic, and higher-order hadronic contributions, respectively. The difference between the experimental and the theoretical values of $a_{\mu}, \Delta\left(a_{\mu}\right)$, is

$$
\Delta\left(a_{\mu}\right)=a_{\mu}^{\exp }-a_{\mu}^{\mathrm{SM}}=288(63)(49) \times 10^{-11},
$$

corresponding to a $\sim 3.6 \sigma$ deviation from the SM prediction [32].

The impressive accuracy of the theoretical and experimental results renders $a_{\mu}$ a high precision observable extremely sensitive to physics beyond the SM. Explaining this deviation has motivated extensive studies - not only taking into account the many possible (higher order) SM corrections, but also exploring new physics contributions capable of saturating the observed discrepancy [60]. Singlet extensions of the SM have been considered to address the $(g-2)_{\mu}$ discrepancy; for instance, standard seesaws have been investigated [61], low-scale supersymmetric seesaw models (including right-handed neutrino superfields) [62], as well as $B-L$ (Inverse Seesaw) extensions of the SM [63], among many others.

Regarding the anomalous magnetic moment of the tau, the experimental precision [64] is very poor compared with the theoretical calculation error [65],

$$
\begin{gathered}
a_{\tau}^{\mathrm{SM}}=117721(5) \times 10^{-8} \\
-0.052<a_{\tau}^{\exp }<0.013
\end{gathered}
$$

so that unfortunately this observable cannot in general be used to infer any useful information on possible new physics contributions.

In our work we address the impact of the extra sterile neutrinos on the anomalous magnetic moment of the muon, while considering their contribution to the $(g-2)$ of the electron as a potential new constraint in our analysis. In the SM extended by sterile fermionic states, the only new contribution to the anomalous magnetic moment of leptons arises from the diagram of figure 1 , where $\nu_{i}$ denotes the neutral fermions, including the new sterile states. In the presence of the latter, the $W-\nu$ loop provides the following contribution

$$
a_{\mu}^{\nu}=\frac{G_{F}}{\sqrt{2}} \frac{m_{\mu}^{2}}{8 \pi^{2}} \sum_{i=1}^{n_{\nu}} U_{\mu i}^{*} U_{\mu i} f\left(x_{\nu_{i}}\right),
$$


where $x_{\nu_{i}}=\left(m_{\nu_{i}} / M_{W}\right)^{2}\left(n_{\nu}\right.$ being the number of neutrino mass states, including the sterile ones) and $f(x)$ is given by

$$
f(x)=\frac{10-43 x+78 x^{2}-49 x^{3}+4 x^{4}+18 x^{3} \ln (x)}{3(1-x)^{4}} .
$$

The above result is in good agreement with the corresponding one of [63], derived in the framework of a $B-L$ extension of the SM.

\section{Sterile neutrino extensions of the SM}

In order to account for neutrino masses and mixings, many extensions of the SM call upon the introduction of right-handed neutrinos (giving rise to a Dirac mass term for the neutrinos) and/or other new particles. We consider a minimal extension of the SM, an ad-hoc effective model (the " $3+1$ " effective model) as a representative case. There are several well-motivated neutrino mass generation models relying on the introduction of sterile states, and in this work we will illustrate them through an example of a realisation of a low-scale seesaw mechanism, the Inverse Seesaw mechanism.

\subsection{Constraints on sterile neutrino models}

In the framework of the SM extended by sterile fermion states, which have a non-vanishing mixing to the active neutrinos, the leptonic charged currents are modified as

$$
-\mathcal{L}_{\mathrm{cc}}=\frac{g}{\sqrt{2}} U^{j i} \bar{\ell}_{j} \gamma^{\mu} P_{L} \nu_{i} W_{\mu}^{-}+\text {c.c. }
$$

where $U$ is the leptonic mixing matrix, $i=1, \ldots, n_{\nu}$ denotes the physical neutrino states and $j=1, \ldots, 3$ the flavour of the charged leptons. In the case of three neutrino generations, $U$ corresponds to the (unitary) PMNS matrix, $U_{\text {PMNS }}$. For $n_{\nu}>3$, the mixing between the left-handed leptons, which we will subsequently denote by $\tilde{U}_{\text {PMNS }}$, corresponds to a $3 \times 3$ block of $U$. One can parametrise the $\tilde{U}_{\text {PMNS }}$ mixing matrix as [66]

$$
U_{\mathrm{PMNS}} \rightarrow \tilde{U}_{\mathrm{PMNS}}=(\mathbb{1}-\eta) U_{\mathrm{PMNS}}
$$

where the matrix $\eta$ encodes the deviation of $\tilde{U}_{\text {PMNS }}$ from unitarity $[12,13]$, due to the presence of extra fermion states.

Many observables will be sensitive to the active-sterile mixings, and their current experimental values (or bounds) will thus constrain such SM extensions. As already mentioned, one can have LFV and LFU violating observables, bounds from laboratory and collider searches, among others. Furthermore, certain sterile mass regimes and active-sterile mixing angles are also strongly constrained by cosmological observations.

In what follows we proceed to discuss the most relevant constraints on models with sterile fermions. 
Neutrino oscillation data: the first and most important constraint to any model of massive neutrinos is to comply with $\nu$-oscillation data [37-41]. In our analysis we will consider both normal and inverted hierarchies for the light neutrino spectrum [37]; the corresponding best-fit intervals in the case of normal hierarchy (NH) $\operatorname{are}^{2}$

$$
\begin{aligned}
& \sin ^{2} \theta_{12}=0.32, \\
& \sin ^{2} \theta_{23}=0.427, \\
& \sin ^{2} \theta_{13}=0.0246, \\
& \Delta m_{21}^{2}=7.62 \times 10^{-5} \mathrm{eV}^{2}, \\
& \left|\Delta m_{31}^{2}\right|=2.55 \times 10^{-3} \mathrm{eV}^{2},
\end{aligned}
$$

whereas for an inverted mass hierarchy (IH) the values are

$$
\begin{aligned}
& \sin ^{2} \theta_{12}=0.32, \\
& \sin ^{2} \theta_{23}=0.6, \\
& \Delta m_{21}^{2}=7.62 \times 10^{-5} \mathrm{eV}^{2}, \\
& \left|\Delta m_{31}^{2}\right|=2.43 \times 10^{-3} \mathrm{eV}^{2} \text {. } \\
& \sin ^{2} \theta_{13}=0.025,
\end{aligned}
$$

The value of the CP violating Dirac phase $\delta$ is still undetermined, although the complementarity of accelerator and reactor neutrino data starts reflecting in a better sensitivity to $\delta[40,67]$ (and to the hierarchy of the light neutrino spectrum).

It is worth noticing that the $\mathrm{CP}$ violating phases of the $U_{\mathrm{PMNS}}$, as well as the possible new phases that will be present in extensions of the SM by sterile neutrinos, will also contribute to the electric dipole moments (EDM) of leptons; however, in these minimal extensions, lepton EDMs would only receive contributions at the 2-loop level. We do not address these $\mathrm{CP}$ violating $(\mathrm{CPV})$ observables in the present analysis.

Unitarity constraints: the introduction of fermionic sterile states can give rise to nonstandard neutrino interactions with matter. Bounds on the non-unitarity of the matrix $\eta$ (cf. eq. (3.2)), have been derived in [43] by means of an effective theory approach. We apply these bounds in our numerical analysis in the regimes for which the latter approach is valid, generically for sterile masses above the GeV, but below the EW scale (at least in extensions calling for more than one sterile state).

Electroweak precision data: the addition of (fermion) singlets to the SM with a sizeable active-sterile mixing can affect electroweak precision observables at tree-level (charged currents) and at higher order as well. In particular, the non-unitarity of the active neutrino mixing matrix, eq. (3.2), implies that the couplings of the active neutrinos to the $Z$ and $W$ bosons are suppressed with respect to their SM values. In the presence of singlet neutrinos, electroweak precision constraints were first addressed in [45] with an effective approach (therefore valid only for multi-TeV singlet states). More recently, the effects of the sterile neutrinos on the invisible $Z$-decay width have been discussed in $[22,23,46]$, and it has been shown that $\Gamma(Z \rightarrow \nu \nu)$ can be reduced with respect to the SM prediction. Complying with LEP results on $\Gamma(Z \rightarrow \nu \nu)$ [32] also constrains these sterile neutrino extensions.

LHC constraints: the new interactions in the leptonic sector can also alter the Higgs boson phenomenology, since the presence of a new decay channel for the Higgs boson, with heavy neutrinos in the final state, can enlarge the total Higgs decay width, thus lowering the predicted SM decay branching ratios. Therefore, constraints on sterile neutrinos are

\footnotetext{
${ }^{2}$ We take the local minimum in the first octant, in agreement with $[38,40]$.
} 
also derived from Higgs decays. LHC data already allows to constrain regimes where the sterile states are below the Higgs mass, due to the potential Higgs decays to an active and a heavier, mostly sterile, neutrino. In our analysis we apply the constraints derived in $[24,25,27,28]$.

Leptonic and semileptonic meson decays: further constraints arise from decays of pseudoscalar mesons $K, D, D_{s}$ and $B$, with one or two neutrinos in the final state (see $[68,69]$ for kaon decays, $[70,71]$ for $D$ and $D_{s}$ decay rates, and $[72,73]$ for $B$-meson observations). In the framework of the SM extended by sterile neutrinos, these decays have recently been addressed in $[21,22]$. The dominant contributions to these processes arise from tree-level $W$ mediated exchanges (a consequence of the modified vertex $W \ell \nu$ due to the presence of the sterile states). As will be discussed in the following section, among the distinct constraints derived from meson decays, the most severe bounds are due to the violation of lepton universality in leptonic kaon decays, parametrised by $\Delta r_{K}$,

$$
\Delta r_{K} \equiv \frac{R_{K}^{\exp }}{R_{K}^{\mathrm{SM}}}-1 \quad \text { where } \quad R_{K} \equiv \frac{\Gamma\left(K^{+} \rightarrow e^{+} \nu\right)}{\Gamma\left(K^{+} \rightarrow \mu^{+} \nu\right)},
$$

and whose current value (comparison of theoretical analyses [74, 75] with the recent measurements from the NA62 collaboration [68]) is

$$
\Delta r_{K}=(4 \pm 4) \times 10^{-3} .
$$

This observable can receive significant contributions from the sterile states, due to the new phase space factors and as a result of deviations from unitarity, when the sterile mixings to the active neutrinos are sizeable $[18,19,21,22]$.

Laboratory searches: robust laboratory bounds on the sterile neutrino masses and their mixings with the active ones can be inferred from negative searches for monochromatic lines in the spectrum of muons from $\pi^{ \pm} \rightarrow \mu^{ \pm} \nu$ decays [10, 76]. The absence of such a signal imposes stringent limits for sterile neutrinos with masses in the $\mathrm{MeV}-\mathrm{GeV}$ range.

Lepton flavour violation: non-negligible active-sterile mixings will affect charged lepton violating (cLFV) processes, leading to rates potentially larger than current bounds, through the enlarged leptonic mixing matrix. The most stringent bound on sterile neutrinos from cLFV processes [14, 15] comes from the search for the radiative $\mu \rightarrow e \gamma$ decay [77].

Neutrinoless double beta decay: the introduction of singlet neutrinos with Majorana masses allows for new processes like LNV interactions. Among these, neutrinoless double beta decay remains the most important one. This process is being actively searched for by several experiments, by means of the best performing detector techniques: among others, GERDA [48], EXO-200 [78, 79] and KamLAND-ZEN [80], have all set strong bounds on the effective mass, $\left|m_{e e}\right|$, to which the amplitude of $0 \nu 2 \beta$ process is proportional. The sensitivities of the current experiments put a limit on the effective neutrino Majorana mass in the range

$$
\left|m_{e e}\right| \lesssim 140 \mathrm{meV}-700 \mathrm{meV}
$$




\begin{tabular}{|l|l|c|}
\hline Experiment & Ref. & $\left|m_{e e}\right|(\mathrm{eV})$ \\
\hline EXO-200 (4 yr) & {$[78,79]$} & $0.075-0.2$ \\
nEXO (5 yr) & {$[81]$} & $0.012-0.029$ \\
nEXO (5 yr + 5 yr w/ Ba tagging) & {$[81]$} & $0.005-0.011$ \\
KamLAND-Zen (300 kg, 3 yr) & {$[80]$} & $0.045-0.11$ \\
GERDA phase II & {$[48]$} & $0.09-0.29$ \\
CUORE (5 yr) & {$[82,83]$} & $0.051-0.133$ \\
SNO+ & {$[84]$} & $0.07-0.14$ \\
SuperNEMO & {$[85]$} & $0.05-0.15$ \\
NEXT & {$[86,87]$} & $0.03-0.1$ \\
MAJORANA demo. & {$[88]$} & $0.06-0.17$ \\
\hline
\end{tabular}

Table 1. Future sensitivity of several $0 \nu 2 \beta$ experiments.

In table 1 , we summarise the future sensitivity of ongoing and planned $0 \nu 2 \beta$ experiments. This observable will be addressed in detail when we discuss each of the sterile neutrino models explored in our study.

Cosmological bounds: sterile neutrinos with a mass below the $\mathrm{TeV}$ are subject to strong constraints from a number of cosmological observations [10, 47]. The sterile states play an important rôle in cosmology and astrophysics, in particular in Big Bang Nucleosynthesis and Large Scale Structure formation. Moreover, a sterile neutrino with a mass $\sim \mathrm{keV}$ may be a viable dark matter candidate, for instance offering a possible explanation to the observed X-ray line in cluster galaxy spectra at $\sim 3.5 \mathrm{keV}[89,90]$, to the origin of pulsar kicks, or even to the baryon asymmetry of the Universe (for a review see [11]).

These cosmological limits are in general derived by assuming the minimal possible abundance (in agreement with neutrino oscillations) of sterile neutrinos in halos consistent with standard cosmology. Nevertheless, as argued in [91], the possibility of a non-standard cosmology with a very low reheating temperature, or a scenario where the sterile neutrinos couple to a dark sector [92], could allow to evade some of the above bounds. For this reason, and aiming at being conservative, in our numerical study we will allow for the violation of these cosmological bounds in some scenarios, explicitly stating it.

\subsection{Effective SM extension: "3+1" model}

A first approach to address the impact of sterile fermions on the magnetic moments of leptons is to consider a minimal effective model with three light active neutrinos and one extra sterile Majorana neutrino. In this approach no assumption is made concerning the underlying mechanism responsible for the origin of neutrino masses and mixings. The extension of the SM by the extra state introduces additional degrees of freedom: its mass $m_{4}$, three new (active-sterile) mixing angles $\theta_{i 4}$, two new (Dirac) CP violating phases and one extra Majorana phase.

Concerning the anomalous lepton magnetic moments, the sum in eq. (2.9) extends to $n_{\nu}=4$, while the effective neutrino mass $m_{e e}$, determining the amplitude of the neutrinoless 
double beta decay rate, is given by [93]:

$$
m_{e e} \simeq \sum_{i=1}^{4} U_{e i}^{2} p^{2} \frac{m_{i}}{p^{2}-m_{i}^{2}} \simeq\left(\sum_{i=1}^{3} U_{e i}^{2} m_{\nu_{i}}\right)+p^{2} U_{e 4}^{2} \frac{m_{4}}{p^{2}-m_{4}^{2}},
$$

where $p^{2} \simeq-(100 \mathrm{MeV})^{2}$ is the virtual momentum of the neutrino (an average estimate over different values depending on the decaying nucleus).

\subsection{The inverse seesaw scenario}

In order to investigate the impact of the sterile neutrinos on the lepton magnetic moments in the concrete framework of a neutrino mass generation mechanism, we have considered the Inverse Seesaw mechanism [36]. The ISS scenario is an appealing extension of the SM, which allows to accommodate neutrino data with natural values of the Yukawa couplings for a comparatively low seesaw scale. In turn, this offers the possibility of having sizeable mixings between the active neutrinos and the additional sterile states, thus rendering the model phenomenologically rich.

The ISS requires the introduction of $n_{R} \geq 2$ generations ${ }^{3}$ of right-handed (RH) neutrinos $\nu_{R}$ and $n_{X}$ generations of extra SU(2) singlets fermions $X$ (such that $n_{R}+n_{X}=N_{s}$ ), both with lepton number $L=+1$ [36]. In our analysis we will consider a realisation with $n_{R}=n_{X}=3$.

In the ISS, the SM Lagrangian is extended as

$$
\mathcal{L}_{\mathrm{ISS}}=\mathcal{L}_{\mathrm{SM}}-Y_{i j}^{\nu} \bar{\nu}_{R i} \tilde{H}^{\dagger} L_{j}-M_{R i j} \bar{\nu}_{R i} X_{j}-\frac{1}{2} \mu_{X i j} \bar{X}_{i}^{c} X_{j}+\text { h.c. },
$$

where $i, j=1,2,3$ are generation indices and $\tilde{H}=i \sigma_{2} H^{*}$. After EW symmetry breaking, the (symmetric) $9 \times 9$ neutrino mass matrix $\mathcal{M}$ is given in the $\left(\nu_{L}, \nu_{R}^{c}, X\right)^{T}$ basis by

$$
\mathcal{M}=\left(\begin{array}{ccc}
0 & m_{D}^{T} & 0 \\
m_{D} & 0 & M_{R} \\
0 & M_{R}^{T} & \mu_{X}
\end{array}\right)
$$

Notice that $\mathrm{U}(1)_{L}$ (i.e., lepton number) is broken only by the non-zero Majorana mass term $\mu_{X}$, while the Dirac-type right-handed neutrino mass term $M_{R}$ conserves lepton number. In the above, $m_{D}=\frac{1}{\sqrt{2}} Y^{\nu} v$ is the Dirac mass, $v$ being the vacuum expectation value of the SM Higgs boson. Assuming $\mu_{X} \ll m_{D} \ll M_{R}$, the diagonalization of $\mathcal{M}$ leads to an effective Majorana mass matrix for the active (light) neutrinos [95],

$$
m_{\nu} \simeq m_{D}^{T} M_{R}^{T-1} \mu_{X} M_{R}^{-1} m_{D} .
$$

The remaining (mostly) sterile states form nearly degenerate pseudo-Dirac pairs, with masses

$$
m_{S_{ \pm}}= \pm \sqrt{M_{R}^{2}+m_{D}^{2}}+\frac{M_{R}^{2} \mu_{X}}{2\left(m_{D}^{2}+M_{R}^{2}\right)} .
$$

\footnotetext{
${ }^{3}$ This lower value is required in order to account for the active neutrino masses and mixings. The most minimal Inverse Seesaw realisation [94] consists in the addition of two right-handed and two sterile neutrinos to the SM content.
} 
For the purpose of our analysis it is useful to define $M=M_{R} \mu_{X}^{-1} M_{R}^{T}$, which is diagonalized by the matrix $D$ as $D M D^{T}=\hat{M}$. The eigenvalues of $M$ are thus the entries of the diagonal matrix $\hat{M}$. It is also convenient to generalize the Casas-Ibarra parametrisation [96], which allows to write the neutrino Yukawa couplings $Y^{\nu}$ as

$$
Y^{\nu}=\frac{\sqrt{2}}{v} D^{\dagger} \sqrt{\hat{M}} R \sqrt{\hat{m}_{\nu}} U_{\mathrm{PMNS}}^{\dagger}
$$

where $\sqrt{\hat{m}_{\nu}}$ is a diagonal matrix containing the square roots of the three light neutrino mass eigenvalues $m_{\nu} . R$ is an arbitrary $3 \times 3$ complex orthogonal matrix, parametrized by 3 complex angles, which encodes the remaining degrees of freedom. (Without loss of generality, we can work in the basis where $M_{R}$ is a real diagonal matrix, as are the charged lepton Yukawa couplings.) The neutrino mass matrix is then diagonalized by a $9 \times 9$ unitary mixing matrix $U$ as $U^{T} \mathcal{M} U=\operatorname{diag}\left(m_{i}\right)$. In the basis where the charged lepton mass matrix is diagonal, the leptonic mixing matrix is given by the rectangular $3 \times 9$ submatrix corresponding to the first three columns of $U$, with the $3 \times 3$ block corresponding to the (non-unitary) ${ }^{4} \tilde{U}_{\text {PMNS }}$.

While in the " $3+1$ " effective model four states (three active and one sterile) contributed to the sum of eq. (2.9), in the ISS the sum includes 9 mass eigenstates, and reflects the possibility of having the additional (mostly) sterile states present in the loop of figure 1 , thus significantly contributing to the anomalous lepton moments.

Concerning $0 \nu 2 \beta$ decays, the new sterile states will also contribute: their spectrum corresponding to three pseudo-Dirac pairs, cf. eq. (3.12), the effective neutrino mass $m_{e e}$ is now given by

$$
\begin{aligned}
m_{e e} \simeq & \sum_{i=1}^{9} U_{e i}^{2} p^{2} \frac{m_{i}}{p^{2}-m_{i}^{2}} \simeq\left(\sum_{i=1}^{3} U_{e i}^{2} m_{\nu_{i}}\right)+ \\
& +p^{2}\left(-U_{e 4}^{2} \frac{\left|m_{4}\right|}{p^{2}-m_{4}^{2}}+U_{e 5}^{2} \frac{\left|m_{5}\right|}{p^{2}-m_{5}^{2}}-U_{e 6}^{2} \frac{\left|m_{6}\right|}{p^{2}-m_{6}^{2}}\right. \\
& \left.\quad+U_{e 7}^{2} \frac{\left|m_{7}\right|}{p^{2}-m_{7}^{2}}-U_{e 8}^{2} \frac{\left|m_{8}\right|}{p^{2}-m_{8}^{2}}+U_{e 9}^{2} \frac{\left|m_{9}\right|}{p^{2}-m_{9}^{2}}\right)
\end{aligned}
$$

with, as before, $p^{2} \simeq-(100 \mathrm{MeV})^{2}$ the virtual momentum of the neutrino.

\section{Analysis and discussion}

The aim of this study is to evaluate the impact of the new sterile states on the leptonic anomalous magnetic moment, as well as on the neutrinoless double beta decay effective mass, for the two models previously discussed - the " $3+1$ " effective model, and the ISS with 3 right-handed neutrinos and 3 extra sterile states. As mentioned in section 3.1, we will apply in each case all relevant constraints. In the different plots illustrating our study, and for the sake of completeness, we will nonetheless show all the solutions, denoting by different shades of grey the points not complying with the various bounds. An exception

\footnotetext{
${ }^{4}$ We refer to [97-99] for earlier studies on non-unitarity effects in the Inverse Seesaw.
} 
concerns the cosmological constraints: as noticed for example in [91], the cosmological bounds could be modified and eventually evaded by considering a non-standard cosmology. Therefore, and throughout the subsequent discussion, we will not discard solutions in disagreement with the cosmological bounds of [10], highlighting them with a distinctive colour scheme, namely with red coloured points.

As a convenient means to illustrate the effect of the new active-sterile mixings (corresponding to a deviation from unitarity of the $\tilde{U}_{\text {PMNS }}$ ) we have introduced the invariant quantity $\tilde{\eta}$, defined as

$$
\tilde{\eta}=1-\left|\operatorname{Det}\left(\tilde{U}_{\mathrm{PMNS}}\right)\right| .
$$

In our analysis we address both cases of normal and inverted hierarchies for the light neutrino spectrum, and we fully explore the parameter space for each of the models considered, including the new (Dirac and Majorana) CP violating phases. As already stated, we do not address the (2-loop) contributions to the leptonic EDMs.

\subsection{Results: "3+1" effective model}

For both $\mathrm{NH}$ and $\mathrm{IH}$ light neutrino spectra, we scan over the sterile neutrino mass in the range

$$
3 \times 10^{-11} \mathrm{GeV} \lesssim m_{4} \lesssim 10^{3} \mathrm{GeV},
$$

and over the active-sterile mixing angles $\theta_{i 4}(i=1,2,3)$, which are randomly taken between 0 and $2 \pi$. We also consider the effect of all CPV phases, which are likewise randomly varied in $[0,2 \pi]$.

Anomalous magnetic moment of the electron: we first consider the potential impact of $\Delta\left(a_{e}\right)$ as an additional constraint on the parameter space of the " $3+1$ " effective extension of the SM. As can be seen on figure 2, which illustrates this observable for the case of a normal hierarchy in the light neutrino spectrum, the new contributions to $\left|\Delta\left(a_{e}\right)\right|$ do augment with increasing deviations from unitarity of the $\tilde{U}_{\text {PMNS }}$ matrix. However, even for the largest values of $\tilde{\eta}$ - which are excluded due to the violation of several bounds (the most important one being neutrino oscillation data) - the " $3+1$ " effective model remains short of the $1 \sigma$ bound. As already mentioned in section 3.1, we display in red the points that are typically disfavoured from standard cosmology arguments (notice that the large $\tilde{\eta}$ regime in general corresponds to this case). The grey-shades correspond to at least: failure to comply with $\nu$-oscillation data, bounds from EW precision data or LHC bounds (light grey); violation of laboratory bounds or constraints from rare leptonic meson decays (grey); confict with bounds from radiative $\mu \rightarrow e \gamma$ decays, neutrinoless double beta decays or invisible $Z$-boson width (dark grey).

Anomalous magnetic moment of the muon: we proceed to investigate whether this minimal extension of the SM with sterile neutrinos can contribute to alleviate the tension between the experimental measurement and the SM prediction for $(g-2)_{\mu}$, as discussed in section 2. On figure 3, we display the contribution of the " $3+1$ " effective model, for the case of a normal hierarchical light neutrino spectrum. Although we do not explicitly 


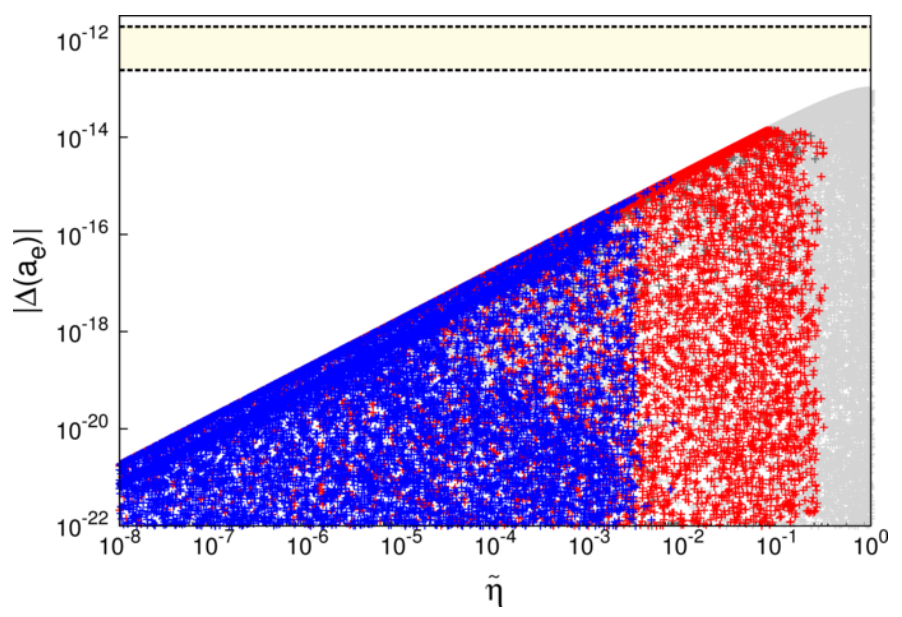

Figure 2. Effective case: anomalous magnetic moment of the electron as a function of $\tilde{\eta}$ (see eq. (4.1)), for a $\mathrm{NH}$ light neutrino spectrum. Blue points are in agreement with cosmological bounds, while the red ones would require considering a non-standard cosmology. In grey we denote points already excluded by other (non-cosmological) bounds (see text for a description of the greyshade scheme). The beige coloured band denotes the $1 \sigma$ interval, cf. eq. (2.3). The underlying scan is described in the text.
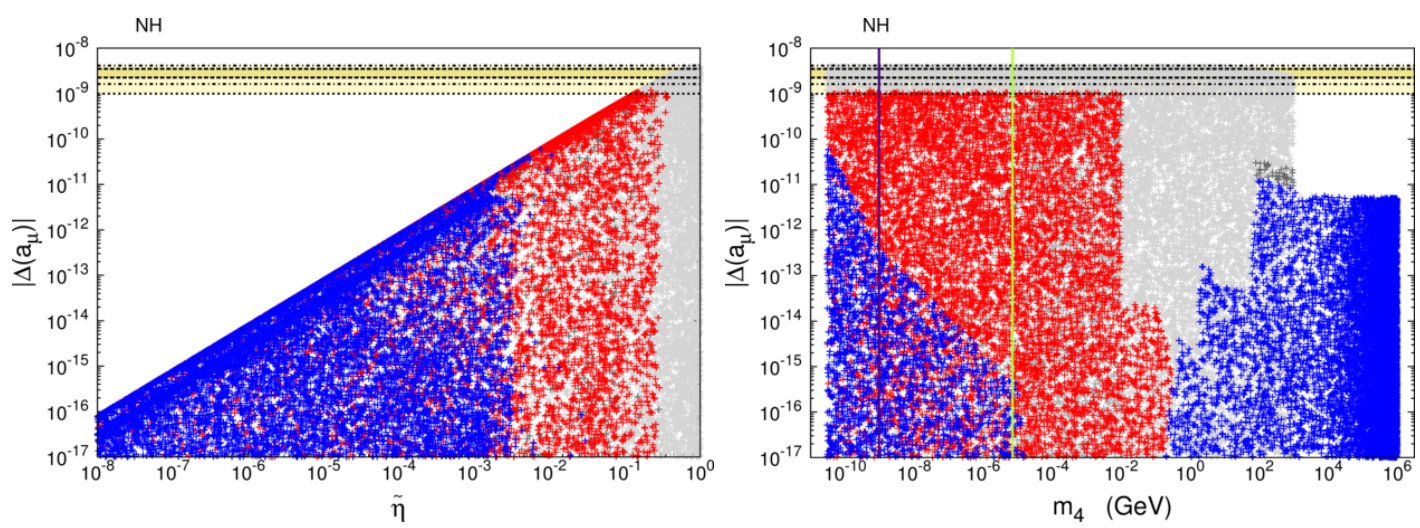

Figure 3. Effective case: anomalous magnetic moment of the muon as a function of $\tilde{\eta}$ (left) and of the mass of the sterile neutrino $m_{4}$ (right), for a NH light neutrino spectrum. The horizontal dashed lines delimit the surfaces corresponding to the $1 \sigma, 2 \sigma$ and $3 \sigma$ intervals for $\left|\Delta\left(a_{\mu}\right)\right|$ (darker to lighter shades of beige), see eq. (2.7). The vertical violet (green) line corresponds to the best fit value of $m_{4}$ which would account for the reactor anomaly (to the $3.5 \mathrm{keV}$ line from decaying warm dark matter). Otherwise, colour scheme as in figure 2.

display them here, phenomenological equivalent results have been obtained for the case of an inverted hierarchy in the active neutrino spectrum.

The left panel of figure 3 reveals that this simple effective extension can account for a contribution capable of slightly alleviating the tension between theory and experiment, albeit close to the viability limit of the model (i.e., large values of $\tilde{\eta}$ ), and only for points disfavoured by cosmological observations. Moreover, these contributions are associated to light sterile states: as can be seen from the right panel of figure 3 , contributions within the $3 \sigma$ interval correspond to $m_{4} \lesssim 10^{-2} \mathrm{GeV}$. The two vertical lines of the right panel 

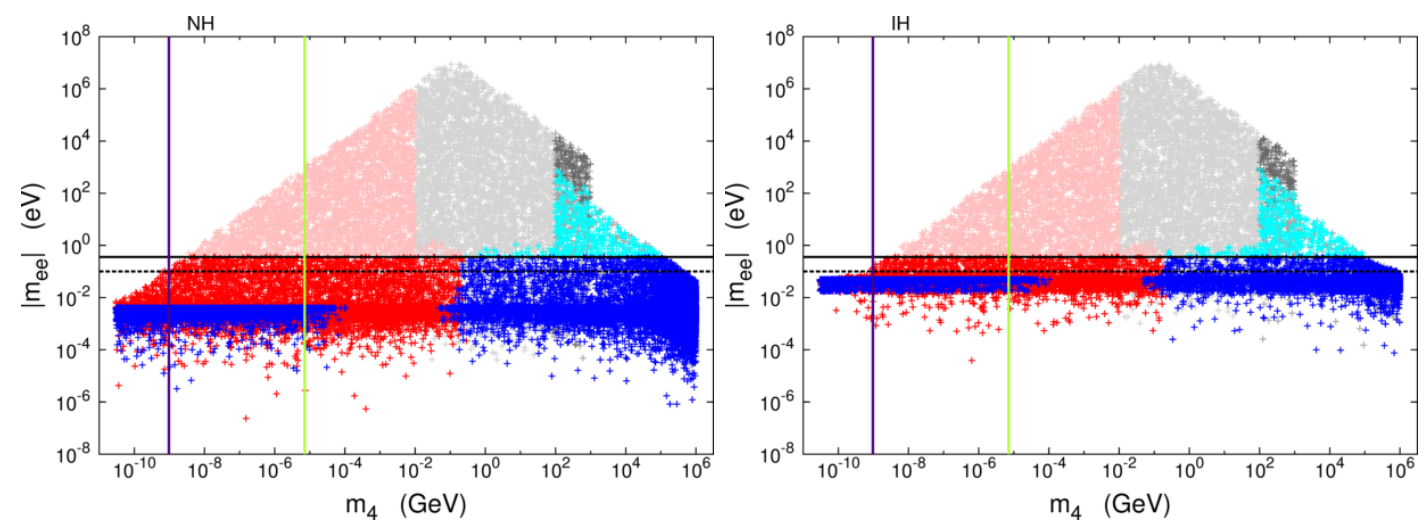

Figure 4. Effective case: effective mass, $\left|m_{e e}\right|$ (in eV), as a function of the mass of the sterile neutrino $m_{4}$, for NH (left) and IH (right) light neutrino spectra. The solid (dashed) horizontal line denotes the current bound (expected future sensitivity), see table 1, while the vertical lines correspond to the values of $m_{4}$ which would account for the reactor anomaly and explain the $3.5 \mathrm{keV}$ line from decaying warm dark matter. Blue points are in agreement with cosmological bounds, while the red ones would require considering a non-standard cosmology. Cyan/pink respectively correspond to cosmologically favoured/disfavoured points which are excluded due to conflict with bounds from $0 \nu 2 \beta$ decays. In grey we again denote points already excluded by other (non-cosmological) bounds.

denote the values of the mostly (lightest) sterile state mass that would allow to address issues strongly motivating these extensions of the SM: (i) $m_{4} \simeq 1 \mathrm{eV}$, the best fit value for a sterile state accounting for the reactor anomaly [100]; (ii) $m_{4} \sim 7 \mathrm{keV}$, as required to explain the $3.5 \mathrm{keV}$ line in the X-ray spectra of galaxy clusters from the decay of a warm dark matter candidate [89, 90].

We stress that this is a simple, minimal extension and that the analysis relies on a first order (one-loop) computation of the observable. A more complete computation, including higher order (leptonic and hadronic) contributions could further reduce the present discrepancy.

Anomalous magnetic moment of the tau: we have also considered the contribution of the " $3+1$ " effective model to the anomalous magnetic moment of the tau lepton. Throughout the investigated parameter space, we found that the new contributions saturate at

$$
\left|\Delta\left(a_{\tau}\right)\right| \lesssim 10^{-5}
$$

and are thus clearly beyond experimental reach.

Neutrinoless double beta decay: as previously mentioned, we also revisit the prospects of the " $3+1$ " effective model regarding $0 \nu 2 \beta$ (constraints on the parameter space, as well as the potential for a detection in the near future). In figure 4 we present the expected ranges for $\left|m_{e e}\right|$ as a function of the sterile mass. The horizontal lines denote current bounds and future sensitivities, in agreement with the discussion in table 1. More precisely, we have considered the current bound of $\left|m_{e e}\right| \lesssim 300 \mathrm{meV}$ [48] also showing a future expected sensitivity of $\left|m_{e e}\right| \lesssim 100 \mathrm{meV}$. 

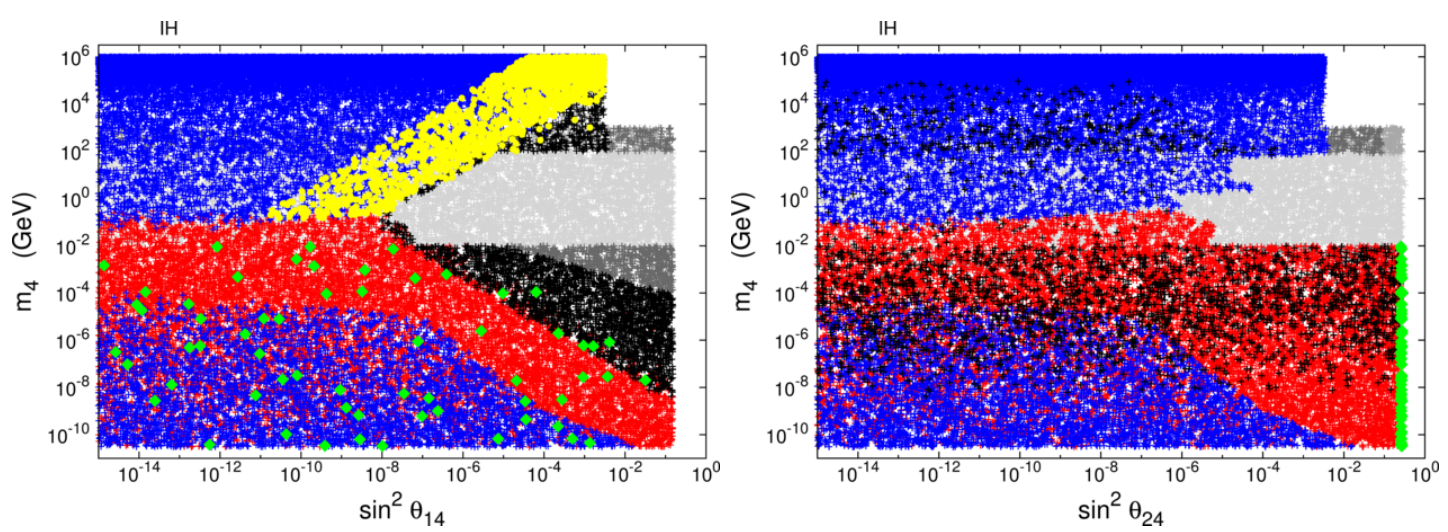

Figure 5. Effective case: parameter space of the sterile state for an IH light neutrino spectrum. On the left, $\left(\sin ^{2} \theta_{14}, m_{4}\right)$ plane, and on the right the $\left(\sin ^{2} \theta_{24}, m_{4}\right)$ one. Blue points are in agreement with cosmological bounds, while the red ones would require considering a non-standard cosmology. Black regions correspond to violating (at least) the $0 \nu 2 \beta$ constraints, while grey ones are excluded due to conflict with other (non-cosmological) bounds. Green diamonds correspond to the points associated to $\left|\Delta\left(a_{\mu}\right)\right|$ within the $3 \sigma$ interval. A yellow band (left panel) further denotes $\left|m_{e e}\right|$ within experimental reach (i.e. $0.05 \mathrm{eV} \lesssim\left|m_{e e}\right| \lesssim 0.1 \mathrm{eV}$ ) for cosmologically allowed points.

As can be seen from figure 4, both hierarchies can lead to significant values of $\left|m_{e e}\right|$, and this observable plays in fact an important rôle in excluding sizeable regions of the " $3+1$ " effective model parameter space. For the heavy mass regime, a future observation would allow to probe both hierarchies, and would correspond to regions of the parameter space compatible with cosmological constraints. For the low mass regime $\left(m_{4} \lesssim 0.1 \mathrm{GeV}\right)$, future experiments can only probe regions that would require considering a non-standard cosmology. Notice however, that for the IH case (which, as expected, is associated to larger values of $\left.\left|m_{e e}\right|\right)$, the low mass regime could be potentially probed by, for instance SNO in its "Phase 2" [84] (we did not explicitly include the corresponding future sensitivity in the plots of figure 4).

It is worth emphasizing here that, under the assumption that sterile neutrinos are present, a signal in $0 \nu 2 \beta$ decay future experiments does not necessarily imply an IH for the light neutrino spectrum.

Leading to both panels of figure 4, we have taken into account non-vanishing values of all (Dirac and Majorana) phases. The CP-conserving (real) limit would translate into similar plots - the only significant difference being a less disperse pattern for the points; we thus refrain from including them here.

To complete the study conducted within this model, we display in figure 5 the " $3+1$ " effective parameter space, in particular the $\left(\sin ^{2} \theta_{i 4}, m_{4}\right)$ planes. We highlight in black regions in which (at least) the $0 \nu 2 \beta$ constraints are violated; those in yellow correspond to cosmologically viable points having $\left|m_{e e}\right|$ within experimental reach (i.e. $0.05 \mathrm{eV} \lesssim\left|m_{e e}\right| \lesssim$ $0.1 \mathrm{eV})$. Green diamonds correspond to the points associated to $\left|\Delta\left(a_{\mu}\right)\right|$ within the $3 \sigma$ interval (although some of these points appear to correspond to regions in agreement with cosmological bounds, we stress that they correspond to cosmologically disfavoured regimes, as explicitly displayed in figure 3 ). 

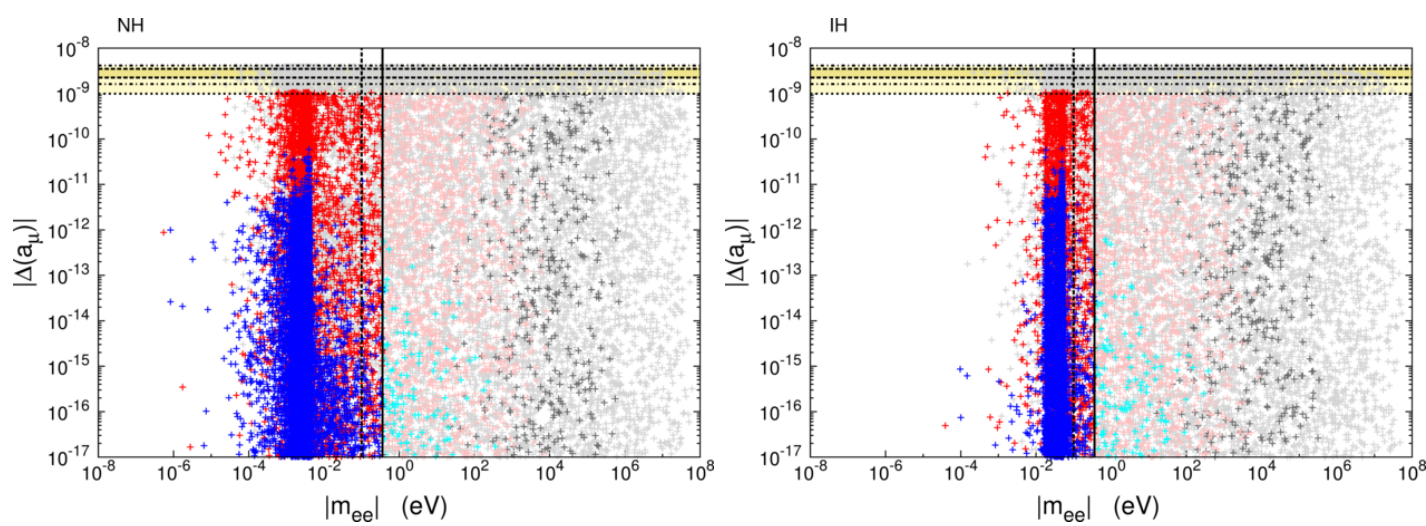

Figure 6. Effective case: summary of the " $3+1$ " effective model prospects regarding its contributions to $\left|\Delta\left(a_{\mu}\right)\right|$ and $0 \nu 2 \beta$ decay. On the left (right) panel, $\mathrm{NH}$ (IH) for the light neutrino spectrum. The horizontal lines denote the $1 \sigma-3 \sigma$ intervals for $\left|\Delta\left(a_{\mu}\right)\right|$, while vertical full (dashed) correspond to the current bounds (future sensitivity) for $\left|m_{e e}\right|$. Colour code as in figure 4.

Figure 5 clearly encodes the phenomenological rôle of the active-sterile mixing angles: it confirms that the largest contributions to $\left|\Delta\left(a_{\mu}\right)\right|$ are, as expected, related to large values ${ }^{5}$ of $\theta_{24}$, while $\theta_{14}$ is the relevant parameter regarding $0 \nu 2 \beta$ decays. As already commented when discussing figure 3 , the largest values for $\left|\Delta\left(a_{\mu}\right)\right|$ correspond to regimes of sizeable sterile-active mixing (as seen from the right panel), pulling the entries of $\tilde{U}_{\text {PMNS }}$ towards the limits of phenomenological and experimental viability. (The analogous analysis of the $\left(\sin ^{2} \theta_{34}, m_{4}\right)$ plane does not provide any new information, and so we do not display it here.) The case of a normal hierarchy in the light neutrino spectrum would lead to similar results for the different $\left(\sin ^{2} \theta_{i 4}, m_{4}\right)$ planes - the exception being the prospects for $0 \nu 2 \beta$ decays (see figure 4$)$; in particular the yellow band in the $\left(\sin ^{2} \theta_{14}, m_{4}\right)$ plane would be significantly narrower.

Summary for the " $3+1$ " effective model: a global overview of the prospects of the " $3+1$ " effective model regarding both $(g-2)_{\mu}$ and neutrinoless double beta decay is presented in figure 6 , for NH and IH light neutrino spectra. Since both observables have already been extensively discussed, we only stress that in the framework of this simple extension of the SM, having $\left|\Delta\left(a_{\mu}\right)\right|$ within the $3 \sigma$ interval and a possible observation of $0 \nu 2 \beta$ decay in the next generation of dedicated facilities requires invoking a non-standard cosmology.

\subsection{Results: inverse seesaw scenario}

In the ISS, the numerical contributions to the studied observables are derived through the following general scan: leading to the construction of the $9 \times 9$ mass matrix in eq. (3.10), the moduli of the entries of the matrices $M_{R}$ and $\mu_{X}$ are randomly taken to lie on the intervals $0.1 \mathrm{MeV} \lesssim\left(M_{R}\right)_{i} \lesssim 10^{6} \mathrm{GeV}$ and $0.01 \mathrm{eV} \lesssim\left(\mu_{X}\right)_{i j} \lesssim 1 \mathrm{MeV}$, with complex entries for the lepton number violating matrix $\mu_{X}$; we also take complex angles for the arbitrary $R$ matrix, randomly varying their values in the interval $[0,2 \pi]$. The modified Casas-Ibarra

\footnotetext{
${ }^{5}$ Notice that in this plot values of $\sin ^{2} \theta_{14} \simeq 1$, which are excluded due to conflict with $\nu$-oscillation data, are not displayed.
} 


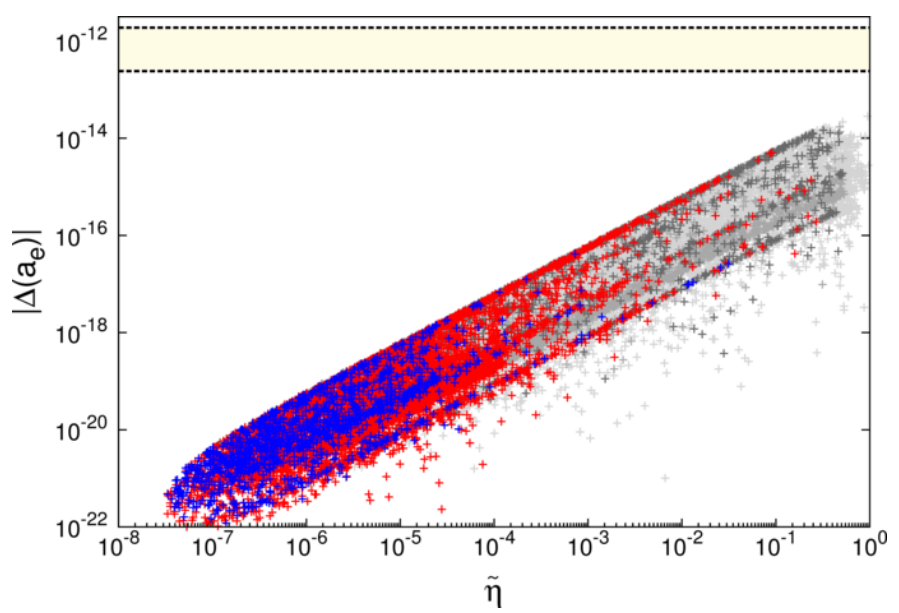

Figure 7. ISS: anomalous magnetic moment of the electron as a function of $\tilde{\eta}$, for a NH light neutrino spectrum. Line and colour code as in figure 2 (see text for a description of the grey-shade scheme).

parametrization for $Y^{\nu}$, eq. (3.13), ensures that constraints from neutrino oscillation data are satisfied. We use as input the best-fit values of the global analysis of [37], for each light neutrino hierarchy: $\mathrm{NH}$ and $\mathrm{IH}$.

Anomalous magnetic moment of the electron: as done for the " $3+1$ " effective model, we begin the analysis of the ISS case by investigating the possible constraints arising from $\left|\Delta\left(a_{e}\right)\right|$. Similarly we find that despite having the new contributions to $\left|\Delta\left(a_{e}\right)\right|$ steadily augmenting with $\tilde{\eta}$, the ISS parameter space is not constrained by the precise determination of this observable. This is illustrated for the case of a $\mathrm{NH}$ in the light neutrino spectrum in figure 7. (The predictions for the IH case are similar.)

Figure 7 also provides a first illustration of some relevant features of the ISS parameter space: ${ }^{6}$ regimes corresponding to a significant deviation from unitarity of the $\tilde{U}_{\text {PMNS }}$ matrix (i.e. large $\tilde{\eta}$ ) are subject to strong experimental constraints, which exclude important parts of the parameter space. The grey-shades in figure 7 correspond to, at least: failure to comply with $\nu$-oscillation data (light grey); violation of unitarity constraints, bounds from EW precision data, laboratory and LHC bounds, rare decays such as leptonic meson decays or radiative $\mu \rightarrow e \gamma$ decays (grey); neutrinoless double beta decays or invisible $Z$-boson width (dark grey). In particular $\nu$-oscillation data and rare kaon decay bounds $\left(\Delta r_{K}\right)$ are the most relevant ones for the ISS parameter space. Finally, and with the exception of a few (isolated) points, notice that compatibility with cosmological constraints is in general obtained only for the regime of small $\tilde{\eta}$.

Anomalous magnetic moment of the muon: the contribution of the ISS concerning the anomalous magnetic moment of the muon is illustrated, for both light neutrino spectrum hierarchies, in figure 8. As can be seen, only a very small fraction of the points

\footnotetext{
${ }^{6}$ The comparative smaller dispersion of the points in the ISS case is merely due to having an underlying theoretical framework relating the active, right-handed and sterile neutrinos, so that the different masses and mixings are not independent degrees of freedom.
} 

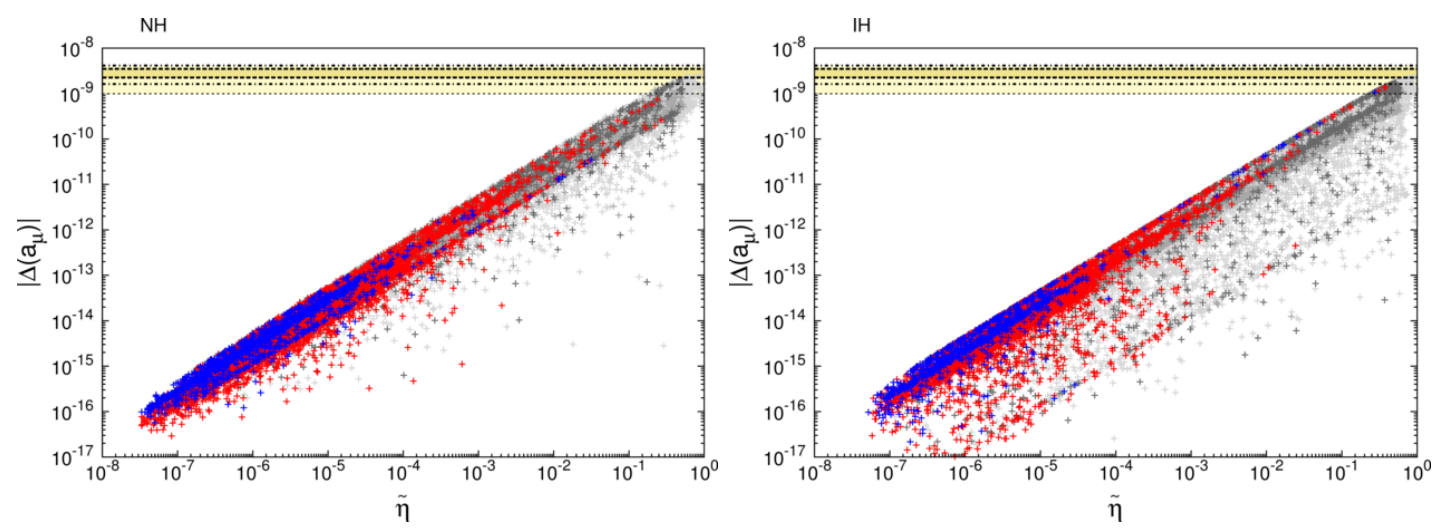

Figure 8. ISS case: anomalous magnetic moment of the muon as a function of $\tilde{\eta}$ for a NH (left) and an IH (right) light neutrino spectrum. Colour scheme as in figure 3 (see text for a description of the grey-shade scheme).

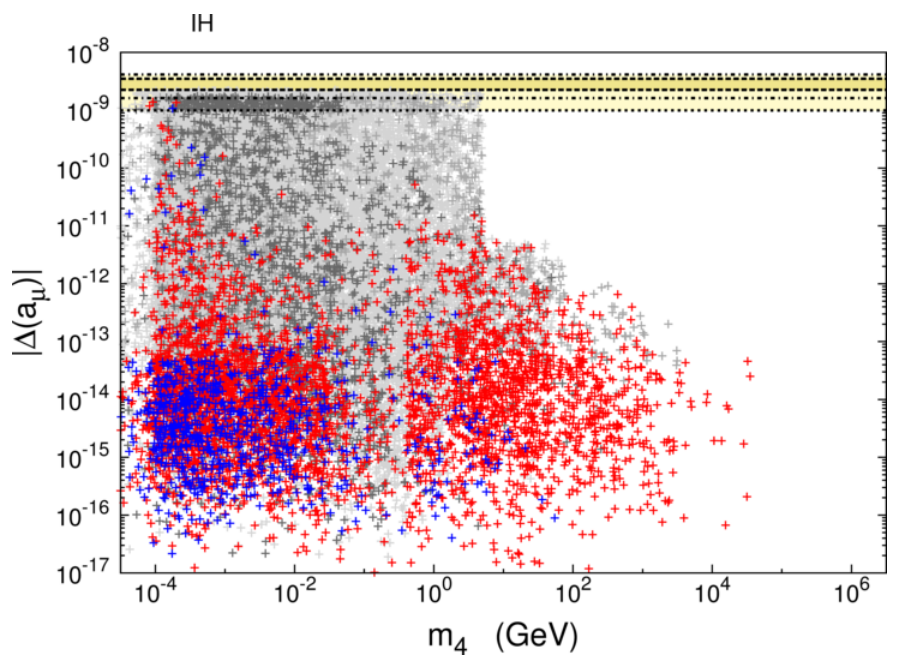

Figure 9. ISS: anomalous magnetic moment of the muon as a function of the mass of the lightest, mostly sterile state $\left(m_{4}\right)$, for an IH light neutrino spectrum. Colour scheme as in figure 3 .

succeeds in providing a contribution within the $3 \sigma$ interval for $\left|\Delta\left(a_{\mu}\right)\right|$, and only for the IH case (interestingly, a few compatible with cosmological bounds). We notice that a much larger set would be within the $3 \sigma$ (even the $2 \sigma$ ) interval, for both hierarchies, but these have been excluded as they lead to excessively large values of $\Delta r_{K}$ (see eq. (3.6)).

We display in figure 9 the ISS contributions to the anomalous magnetic moment of the muon as a function of the lightest (mostly) sterile state mass, ${ }^{7} m_{4}$, as the lighter states provide the dominant contribution to this observable (confirmed from eq. (2.9)). As can be inferred from this figure, accommodating at the $3 \sigma$ level the discrepancy in $(g-2)_{\mu}$ requires that at least one pseudo-Dirac state (pair) be very light, with a mass around $0.1 \mathrm{MeV}$.

\footnotetext{
${ }^{7}$ Contrary to the " $3+1$ " effective model analysis, here we do not include the vertical lines, as this realisation cannot address the corresponding observations. However, a different ISS realisation has been recently shown to be capable of accounting for the $\sim 3.5 \mathrm{keV}$ line in the X-ray spectra of galaxy clusters [101].
} 
Anomalous magnetic moment of the tau: such as it occurred for the " $3+1$ " effective model, the ISS can only account for an extremely small contribution to the anomalous magnetic moment of the tau lepton,

$$
\left|\Delta\left(a_{\tau}\right)\right| \lesssim 10^{-7}
$$

also well beyond experimental reach.

Neutrinoless double beta decay: the predictions of the present ISS realisation, with three right-handed and three sterile neutrino states, are displayed in figure 10, as a function of the average of the absolute masses of the mostly sterile states,

$$
\left\langle m_{4-9}\right\rangle=\sum_{i=4 \ldots 9} \frac{1}{6}\left|m_{i}\right|
$$

for both hierarchies of the light neutrino spectrum. We take this average to illustrate our results as it provides a crude, yet efficient, means to define the "heavy" and "light" mass regimes for the sterile states (as a whole). Leading to both panels, we have conducted a thorough exploration of the impact of the CPV phases of the ISS model (see scan description in the beginning of this section).

The shape of the ISS contributions to $\left|m_{e e}\right|$ is very different from that encountered in the analysis of the " $3+1$ " effective model. This is due to the extended spectrum which, as discussed, is composed of three pseudo-Dirac pairs, the mass difference in each pair of $\mathcal{O}\left(\mu_{X}\right)$, and with each element in a pair accounting for contributions of opposite sign (as can be seen from eq. (3.14)). In the absence of CPV phases, these contributions indeed cancel out to a good approximation, the maximum value saturating around what would be expected from the SM extended by three light active Majorana neutrinos (accounting for $\nu$ oscillation data) - this can be inferred from figure 11, where we plot the CP conserving case (all Majorana and Dirac phases set to zero) for the IH case. In this CP conserving limit, one verifies that $0 \nu 2 \beta$ bounds hardly constrain the ISS parameter space, and that a near future $0 \nu 2 \beta$ signal could only be accounted for by an ISS realisation requiring a non-standard cosmology. Although we do not display it here, the CP conserving NH case could not account for $\left|m_{e e}\right|$ within experimental reach (even for cosmologically disfavoured points).

The observed behaviour in the above plots has important implications concerning the interpretation of a possible $0 \nu 2 \beta$ signal in the near future since, and contrary to other lowscale models of neutrino mass generation, both hierarchies for the light neutrino spectrum can account for such an observation; a comparatively light sterile spectrum, with an average mass scale between $10^{-3} \mathrm{GeV}$ and $100 \mathrm{GeV}$, can account for such a signal, irrespective of the hierarchy, still complying with cosmological observations. Moreover, it would strongly suggest non-vanishing (Majorana) CPV phases.

Summary for the "ISS": as done for the " $3+1$ " effective model, we summarise the prospects of the ISS concerning $(g-2)_{\mu}$ and neutrinoless double beta decays; an overview, for both $\mathrm{NH}$ and $\mathrm{IH}$ light neutrino spectra, is shown in figure 12. One can conclude that should an ISS mechanism be at the origin of neutrino mass generation, a realisation 

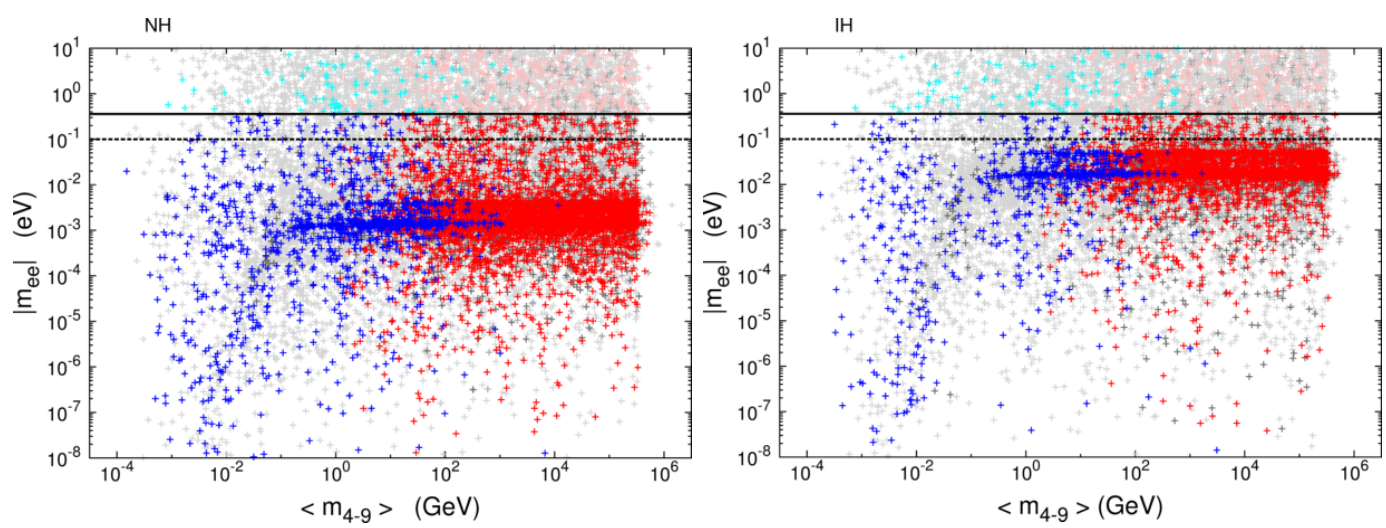

Figure 10. ISS: effective mass $\left|m_{e e}\right|$ (in $\mathrm{eV}$ ) as a function of the average value of the mostly sterile state masses, $\left\langle m_{4-9}\right\rangle$ (see eq. (4.5)), for NH (left) and IH (right) light neutrino spectra. Line and colour scheme as in figure 4.

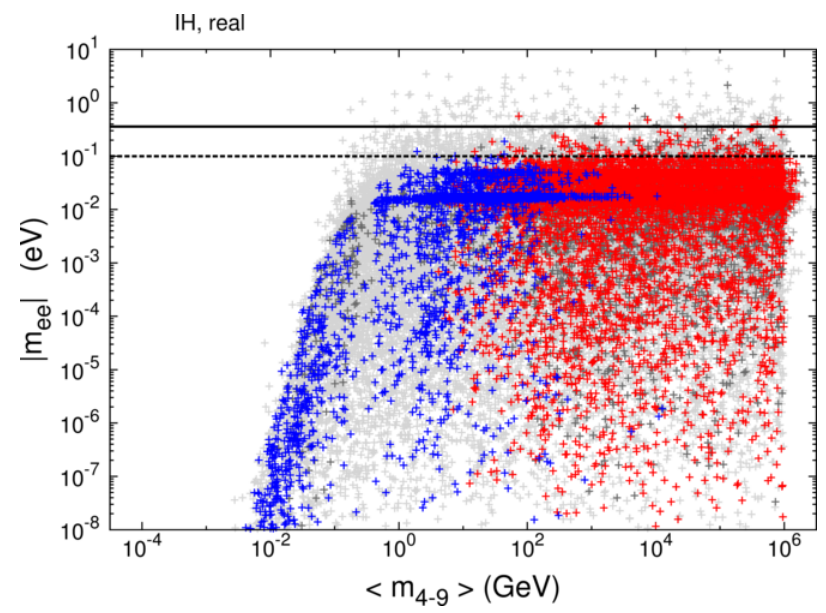

Figure 11. ISS: effective mass $\left|m_{e e}\right|$ (in $\mathrm{eV}$ ) as a function of the average value of the mostly sterile state masses, $\left\langle m_{4-9}\right\rangle$ (see eq. (4.5)), for the CP conserving limit and an IH light neutrino spectrum. Line and colour scheme as in figure 4 .

accounting for a near future observation of a neutrinoless double beta decay signal cannot alleviate the tension in $(g-2)_{\mu}$, not even at the $3 \sigma$ level.

\section{Conclusions}

In this work we investigated the rôle of sterile neutrinos on the (anomalous) magnetic moment of leptons, as well as their contribution to the neutrinoless double beta decay effective mass. We considered minimal extensions of the SM by sterile fermion states. The simplest case is that of an effective construction where, without any assumption on the neutrino mass and mixing generation mechanism, the neutral fermion spectrum contains an additional massive state, which mixes with the active states, leading to an enlarged leptonic mixing matrix. We then focused on a specific well-motivated framework, which 

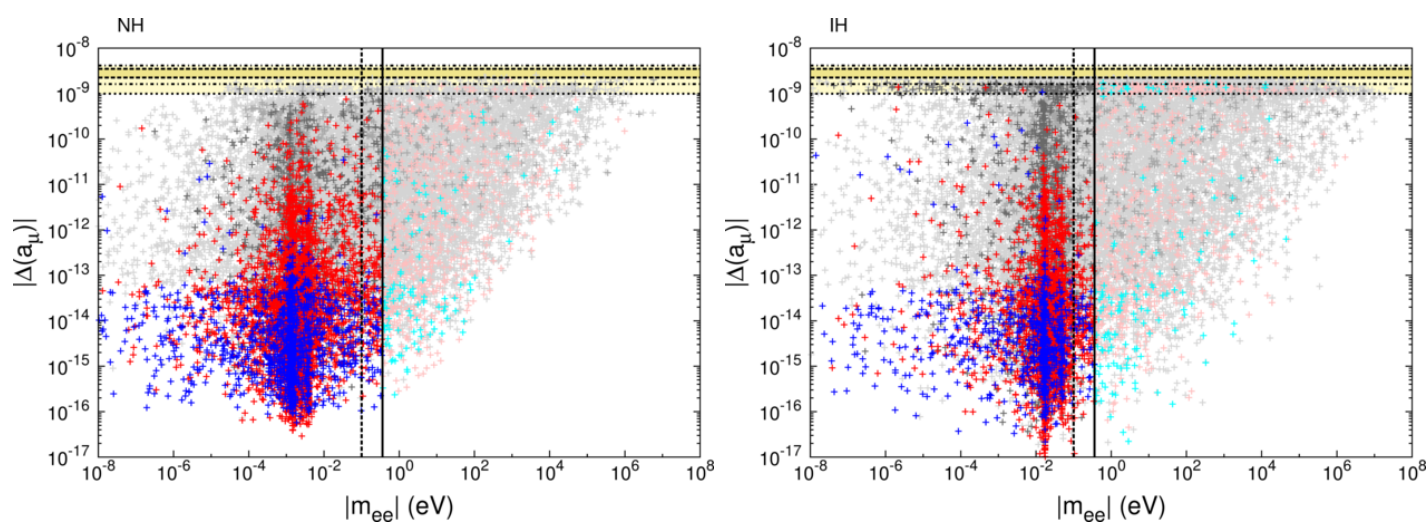

Figure 12. ISS: overview of the prospects regarding the contributions to $\left|\Delta\left(a_{\mu}\right)\right|$ and $0 \nu 2 \beta$ decay. On the left (right) panel, $\mathrm{NH}(\mathrm{IH})$ for the light neutrino spectrum. The horizontal lines denote the $1 \sigma$-3 $\sigma$ intervals for $\left|\Delta\left(a_{\mu}\right)\right|$, while vertical full (dashed) correspond to the current bounds (future sensitivity) for $\left|m_{e e}\right|$. Colour code as in figure 4.

consists in the embedding of an Inverse Seesaw mechanism into the SM, which is extended by three right-handed neutrinos and three sterile states.

Our study reveals that the simple " $3+1$ " effective extension of the SM can account for a contribution capable of alleviating the tension between theory and experiment on $(g-2)_{\mu}$, for large values of $\tilde{\eta}$, and a light sterile state, $m_{4} \lesssim 10^{-2} \mathrm{GeV}$ (although only for points disfavoured by cosmological observations). Regarding $0 \nu 2 \beta$ decays, and in the regime of a heavy sterile mass, a future observation would allow to probe both hierarchies (for a spectrum compatible with cosmological constraints). For the low mass regime $\left(m_{4} \lesssim\right.$ $0.1 \mathrm{GeV}$ ), future $0 \nu 2 \beta$ experiments can only probe regions of the parameter space that would require considering a non-standard cosmology. As shown in our analysis, and under the assumption that sterile neutrinos are present, a signal in $0 \nu 2 \beta$ decay future experiments does not necessarily imply an IH for the light neutrino spectrum. Finally, we have shown that having $\left|\Delta\left(a_{\mu}\right)\right|$ within the $3 \sigma$ interval and a possible observation of $0 \nu 2 \beta$ decay in the next generation of dedicated experiments, requires invoking a non-standard cosmology.

The analysis of the ISS scenario revealed that a contribution within the $3 \sigma$ interval for $\left|\Delta\left(a_{\mu}\right)\right|$ is indeed possible, albeit corresponding to a very small fraction of the phenomenological viable ISS parameter space (and only for the IH case), as the most promising regions are excluded due to excessively large values of $\Delta r_{K}$. Regarding $\left|m_{e e}\right|$, and contrary to other low-scale models of neutrino mass generation, in the considered ISS configuration both hierarchies (IH and NH) can account for an observation in near future facilities. We have also argued that should an ISS mechanism be indeed at the origin of neutrino mass generation, a realisation accounting for a near future observation of a neutrinoless double beta decay signal cannot alleviate the tension in $(g-2)_{\mu}$, not even at the $3 \sigma$ level.

Finally, the contribution to the electron and tau magnetic moments lies in both cases beyond experimental reach. 


\section{Acknowledgments}

The authors acknowledge support from the European Union FP7 ITN INVISIBLES (Marie Curie Actions, PITN-GA-2011-289442). V.D.R. and A.M.T. are grateful for the kind hospitality of the LPT-Orsay, where part of this work was carried out.

Open Access. This article is distributed under the terms of the Creative Commons Attribution License (CC-BY 4.0), which permits any use, distribution and reproduction in any medium, provided the original author(s) and source are credited.

\section{References}

[1] T. Mueller et al., Improved predictions of reactor antineutrino spectra, Phys. Rev. C 83 (2011) 054615 [arXiv:1101.2663] [INSPIRE].

[2] P. Huber, On the determination of anti-neutrino spectra from nuclear reactors, Phys. Rev. C 84 (2011) 024617 [Erratum ibid. C 85 (2012) 029901] [arXiv:1106. 0687] [INSPIRE].

[3] G. Mention et al., The reactor antineutrino anomaly, Phys. Rev. D 83 (2011) 073006 [arXiv:1101.2755] [INSPIRE].

[4] LSND collaboration, A. Aguilar-Arevalo et al., Evidence for neutrino oscillations from the observation of anti-neutrino(electron) appearance in a anti-neutrino(muon) beam, Phys. Rev. D 64 (2001) 112007 [hep-ex/0104049] [INSPIRE].

[5] MiniBoonE collaboration, A.A. Aguilar-Arevalo et al., A Search for electron neutrino appearance at the $\Delta m^{2} \sim 1 e V^{2}$ scale, Phys. Rev. Lett. 98 (2007) 231801 [arXiv: 0704.1500] [INSPIRE].

[6] MiniBoonE collaboration, A.A. Aguilar-Arevalo et al., Event excess in the MiniBooNE search for $\bar{\nu}_{\mu} \rightarrow \bar{\nu}_{e}$ oscillations, Phys. Rev. Lett. 105 (2010) 181801 [arXiv:1007.1150] [INSPIRE].

[7] MiniBoonE collaboration, A.A. Aguilar-Arevalo et al., Improved search for $\bar{\nu}_{\mu} \rightarrow \bar{\nu}_{e}$ oscillations in the MiniBooNE experiment, Phys. Rev. Lett. 110 (2013) 161801 [arXiv: 1207.4809] [INSPIRE].

[8] M.A. Acero, C. Giunti and M. Laveder, Limits on $\nu_{e}$ and $\bar{\nu}_{e}$ disappearance from Gallium and reactor experiments, Phys. Rev. D 78 (2008) 073009 [arXiv:0711.4222] [INSPIRE].

[9] C. Giunti and M. Laveder, Statistical significance of the gallium anomaly, Phys. Rev. C $\mathbf{8 3}$ (2011) 065504 [arXiv: 1006.3244] [INSPIRE].

[10] A. Kusenko, Sterile neutrinos: the dark side of the light fermions, Phys. Rept. 481 (2009) 1 [arXiv:0906.2968] [INSPIRE].

[11] K.N. Abazajian et al., Light sterile neutrinos: a white paper, arXiv:1204.5379 [INSPIRE].

[12] J. Schechter and J.W.F. Valle, Neutrino masses in $\mathrm{SU}(2) \times \mathrm{U}(1)$ theories, Phys. Rev. D 22 (1980) 2227 [INSPIRE].

[13] M. Gronau, C.N. Leung and J.L. Rosner, Extending limits on neutral heavy leptons, Phys. Rev. D 29 (1984) 2539 [INSPIRE].

[14] A. Ilakovac and A. Pilaftsis, Flavor violating charged lepton decays in seesaw-type models, Nucl. Phys. B 437 (1995) 491 [hep-ph/9403398] [INSPIRE]. 
[15] F. Deppisch and J.W.F. Valle, Enhanced lepton flavor violation in the supersymmetric inverse seesaw model, Phys. Rev. D 72 (2005) 036001 [hep-ph/0406040] [INSPIRE].

[16] E. Arganda, M.J. Herrero, X. Marcano and C. Weiland, Imprints of massive ISS neutrinos in LFV Higgs decays, arXiv: 1405.4300 [INSPIRE].

[17] E. Arganda, M.J. Herrero, X. Marcano and C. Weiland, Lepton flavour violating Higgs decays, arXiv:1406.0384 [INSPIRE].

[18] R.E. Shrock, New tests for and bounds on, neutrino masses and lepton mixing, Phys. Lett. B 96 (1980) 159 [INSPIRE].

[19] R.E. Shrock, General theory of weak leptonic and semileptonic decays. 1. Leptonic pseudoscalar meson decays, with associated tests for and bounds on, neutrino masses and lepton mixing, Phys. Rev. D 24 (1981) 1232 [INSPIRE].

[20] E. Nardi, E. Roulet and D. Tommasini, Limits on neutrino mixing with new heavy particles, Phys. Lett. B 327 (1994) 319 [hep-ph/9402224] [INSPIRE].

[21] A. Abada, D. Das, A.M. Teixeira, A. Vicente and C. Weiland, Tree-level lepton universality violation in the presence of sterile neutrinos: impact for $R_{K}$ and $R_{\pi}$, JHEP 02 (2013) 048 [arXiv:1211.3052] [INSPIRE].

[22] A. Abada, A.M. Teixeira, A. Vicente and C. Weiland, Sterile neutrinos in leptonic and semileptonic decays, JHEP 02 (2014) 091 [arXiv: 1311.2830] [INSPIRE].

[23] E. Akhmedov, A. Kartavtsev, M. Lindner, L. Michaels and J. Smirnov, Improving electro-weak fits with TeV-scale sterile neutrinos, JHEP 05 (2013) 081 [arXiv:1302.1872] [INSPIRE].

[24] P.S. Bhupal Dev, R. Franceschini and R.N. Mohapatra, Bounds on TeV seesaw models from LHC Higgs data, Phys. Rev. D 86 (2012) 093010 [arXiv:1207.2756] [INSPIRE].

[25] A. Das, P.S. Bhupal Dev and N. Okada, Direct bounds on electroweak scale pseudo-Dirac neutrinos from $\sqrt{s}=8 \mathrm{TeV}$ LHC data, Phys. Lett. B 735 (2014) 364 [arXiv:1405.0177] [INSPIRE].

[26] A. Das and N. Okada, Inverse seesaw neutrino signatures at the LHC and ILC, Phys. Rev. D 88 (2013) 113001 [arXiv:1207.3734] [INSPIRE].

[27] C.G. Cely, A. Ibarra, E. Molinaro and S.T. Petcov, Higgs decays in the low scale type I see-saw model, Phys. Lett. B 718 (2013) 957 [arXiv:1208.3654] [InSPIRE].

[28] P. Bandyopadhyay, E.J. Chun, H. Okada and J.-C. Park, Higgs signatures in inverse seesaw model at the LHC, JHEP 01 (2013) 079 [arXiv: 1209.4803] [INSPIRE].

[29] F.F. Deppisch, M. Hirsch and H. Pas, Neutrinoless double beta decay and physics beyond the standard model, J. Phys. G 39 (2012) 124007 [arXiv: 1208.0727] [InSPIRE].

[30] M. Chrzaszcz, Searches for $L F V$ and $L N V$ decays at $L H C b$, arXiv:1301.2088 [INSPIRE].

[31] D. Hanneke, S. Fogwell and G. Gabrielse, New measurement of the electron magnetic moment and the fine structure constant, Phys. Rev. Lett. 100 (2008) 120801 [arXiv:0801.1134] [INSPIRE].

[32] Particle Data Group collaboration, J. Beringer et al., Review of particle physics, Phys. Rev. D 86 (2012) 010001 [INSPIRE].

[33] A. Freitas, J. Lykken, S. Kell and S. Westhoff, Testing the muon $g-2$ anomaly at the LHC, JHEP 05 (2014) 145 [arXiv: 1402.7065] [INSPIRE]. 
[34] T. Asaka, S. Blanchet and M. Shaposhnikov, The $\nu M S M$, dark matter and neutrino masses, Phys. Lett. B 631 (2005) 151 [hep-ph/0503065] [INSPIRE].

[35] A. Ibarra, E. Molinaro and S.T. Petcov, TeV scale see-saw mechanisms of neutrino mass generation, the Majorana nature of the heavy singlet neutrinos and $(\beta \beta)_{0 \nu}$-decay, JHEP 09 (2010) 108 [arXiv: 1007.2378] [INSPIRE].

[36] R.N. Mohapatra and J.W.F. Valle, Neutrino mass and baryon number nonconservation in superstring models, Phys. Rev. D 34 (1986) 1642 [INSPIRE].

[37] D.V. Forero, M. Tortola and J.W.F. Valle, Global status of neutrino oscillation parameters after Neutrino-2012, Phys. Rev. D 86 (2012) 073012 [arXiv:1205.4018] [INSPIRE].

[38] G.L. Fogli et al., Global analysis of neutrino masses, mixings and phases: entering the era of leptonic CP-violation searches, Phys. Rev. D 86 (2012) 013012 [arXiv:1205.5254] [INSPIRE].

[39] M.C. Gonzalez-Garcia, M. Maltoni, J. Salvado and T. Schwetz, Global fit to three neutrino mixing: critical look at present precision, JHEP 12 (2012) 123 [arXiv:1209.3023] [INSPIRE].

[40] D.V. Forero, M. Tortola and J.W.F. Valle, Neutrino oscillations refitted, arXiv: 1405.7540 [INSPIRE].

[41] http://www.nu-fit.org/

[42] S. Antusch, C. Biggio, E. Fernandez-Martinez, M.B. Gavela and J. Lopez-Pavon, Unitarity of the leptonic mixing matrix, JHEP 10 (2006) 084 [hep-ph/0607020] [INSPIRE].

[43] S. Antusch, J.P. Baumann and E. Fernandez-Martinez, Non-standard neutrino interactions with matter from physics beyond the standard model, Nucl. Phys. B 810 (2009) 369 [arXiv: 0807.1003] [INSPIRE].

[44] L. Lello and D. Boyanovsky, Searching for sterile neutrinos from $\pi$ and $K$ decays, Phys. Rev. D 87 (2013) 073017 [arXiv: 1208.5559] [INSPIRE].

[45] F. del Aguila, J. de Blas and M. Pérez-Victoria, Effects of new leptons in electroweak precision data, Phys. Rev. D 78 (2008) 013010 [arXiv: 0803.4008] [INSPIRE].

[46] L. Basso, O. Fischer and J.J. van der Bij, Precision tests of unitarity in leptonic mixing, Europhys. Lett. 105 (2014) 11001 [arXiv:1310.2057] [INSPIRE].

[47] A.Y. Smirnov and R. Zukanovich Funchal, Sterile neutrinos: direct mixing effects versus induced mass matrix of active neutrinos, Phys. Rev. D 74 (2006) 013001 [hep-ph/0603009] [INSPIRE].

[48] GERDA collaboration, M. Agostini et al., Results on neutrinoless double- $\beta$ decay of ${ }^{76}$ Ge from phase I of the GERDA experiment, Phys. Rev. Lett. 111 (2013) 122503 [arXiv:1307.4720] [INSPIRE].

[49] T. Aoyama, M. Hayakawa, T. Kinoshita and M. Nio, Revised value of the eighth-order electron g-2, Phys. Rev. Lett. 99 (2007) 110406 [arXiv:0706.3496] [INSPIRE].

[50] T. Aoyama, M. Hayakawa, T. Kinoshita and M. Nio, Tenth-order lepton anomalous magnetic moment: second-order vertex containing two vacuum polarization subdiagrams, one within the other, Phys. Rev. D 78 (2008) 113006 [arXiv:0810.5208] [INSPIRE].

[51] G.F. Giudice, P. Paradisi and M. Passera, Testing new physics with the electron $g-2$, JHEP 11 (2012) 113 [arXiv:1208.6583] [InSPIRE]. 
[52] A. Aboubrahim, T. Ibrahim and P. Nath, Probe of new physics using precision measurement of the electron magnetic moment, arXiv:1403.6448 [INSPIRE].

[53] P.J. Mohr, B.N. Taylor and D.B. Newell, CODATA recommended values of the fundamental physical constants: 2010, Rev. Mod. Phys. 84 (2012) 1527 [arXiv:1203.5425] [INSPIRE].

[54] M. Passera, The standard model prediction of the muon anomalous magnetic moment, J. Phys. G 31 (2005) R75 [hep-ph/0411168] [InSPIRE].

[55] T. Aoyama, M. Hayakawa, T. Kinoshita and M. Nio, Complete tenth-order QED contribution to the muon g-2, Phys. Rev. Lett. 109 (2012) 111808 [arXiv:1205.5370] [INSPIRE].

[56] A. Czarnecki, B. Krause and W.J. Marciano, Electroweak corrections to the muon anomalous magnetic moment, Phys. Rev. Lett. 76 (1996) 3267 [hep-ph/9512369] [INSPIRE].

[57] T. Gribouk and A. Czarnecki, Electroweak interactions and the muon $g-2$ : bosonic two-loop effects, Phys. Rev. D 72 (2005) 053016 [hep-ph/0509205] [INSPIRE].

[58] C. Gnendiger, D. Stöckinger and H. Stöckinger-Kim, The electroweak contributions to $(g-2)_{\mu}$ after the Higgs boson mass measurement, Phys. Rev. D 88 (2013) 053005 [arXiv:1306.5546] [INSPIRE].

[59] M. Davier, A. Hoecker, B. Malaescu and Z. Zhang, Reevaluation of the hadronic contributions to the muon $g-2$ and to $\alpha_{M Z}$, Eur. Phys. J. C 71 (2011) 1515 [Erratum ibid. C 72 (2012) 1874] [arXiv: 1010.4180] [INSPIRE].

[60] A. Czarnecki and W.J. Marciano, The muon anomalous magnetic moment: a harbinger for 'new physics', Phys. Rev. D 64 (2001) 013014 [hep-ph/0102122] [InSPIRE].

[61] C. Biggio, The contribution of fermionic seesaws to the anomalous magnetic moment of leptons, Phys. Lett. B 668 (2008) 378 [arXiv:0806. 2558] [InSPIRE].

[62] A. Ilakovac, A. Pilaftsis and L. Popov, Lepton dipole moments in supersymmetric low-scale seesaw models, Phys. Rev. D 89 (2014) 015001 [arXiv:1308.3633] [INSPIRE].

[63] W. Abdallah, A. Awad, S. Khalil and H. Okada, Muon anomalous magnetic moment and $\mu \rightarrow$ er in B-L model with inverse seesaw, Eur. Phys. J. C 72 (2012) 2108 [arXiv:1105.1047] [INSPIRE].

[64] DELPHI collaboration, J. Abdallah et al., Study of $\tau$-pair production in photon-photon collisions at LEP and limits on the anomalous electromagnetic moments of the $\tau$ lepton, Eur. Phys. J. C 35 (2004) 159 [hep-ex/0406010] [inSPIRE].

[65] S. Eidelman and M. Passera, Theory of the $\tau$ lepton anomalous magnetic moment, Mod. Phys. Lett. A 22 (2007) 159 [hep-ph/0701260] [INSPIRE].

[66] E. Fernandez-Martinez, M.B. Gavela, J. Lopez-Pavon and O. Yasuda, CP-violation from non-unitary leptonic mixing, Phys. Lett. B 649 (2007) 427 [hep-ph/0703098] [INSPIRE].

[67] F. Capozzi et al., Status of three-neutrino oscillation parameters, circa 2013, Phys. Rev. D 89 (2014) 093018 [arXiv: 1312.2878] [INSPIRE].

[68] NA48/2 Collaboration, NA62 collaboration, E. Goudzovski, Kaon programme at CERN: recent results, PoS EPS-HEP2011 (2011) 181 [arXiv:1111.2818] [INSPIRE].

[69] NA62 collaboration, C. Lazzeroni et al., Precision measurement of the ratio of the charged kaon leptonic decay rates, Phys. Lett. B 719 (2013) 326 [arXiv:1212.4012] [InSPIRE]. 
[70] CLEO collaboration, P. Naik et al., Measurement of the pseudoscalar decay constant $f\left(D_{s}\right)$ using $D_{s}^{+} \rightarrow \tau^{+} \nu, \tau^{+} \rightarrow \rho^{+} \bar{\nu}$ decays, Phys. Rev. D 80 (2009) 112004 [arXiv:0910.3602] [INSPIRE].

[71] H.B. Li, Proceedings of $4^{\text {th }}$ international workshop on charm physics (CHARM2010): Beijing, China, October 21-24, 2010, Int. J. Mod. Phys. Conf. Ser. 02 (2011).

[72] BABAr collaboration, B. Aubert et al., A search for $B^{+} \rightarrow \tau^{+} \nu$ with hadronic $B$ tags, Phys. Rev. D 77 (2008) 011107 [arXiv:0708.2260] [InSPIRE].

[73] Belle collaboration, I. Adachi et al., Evidence for $B^{-} \rightarrow \tau^{-} \bar{\nu}_{\tau}$ with a hadronic tagging method using the full data sample of Belle, Phys. Rev. Lett. 110 (2013) 131801 [arXiv: 1208.4678] [INSPIRE].

[74] V. Cirigliano and I. Rosell, Two-loop effective theory analysis of $\pi(K) \rightarrow e \bar{\nu}_{e}[\gamma]$ branching ratios, Phys. Rev. Lett. 99 (2007) 231801 [arXiv:0707.3439] [INSPIRE].

[75] M. Finkemeier, Radiative corrections to $\pi(l 2)$ and K(l2) decays, Phys. Lett. B 387 (1996) 391 [hep-ph/9505434] [INSPIRE].

[76] A. Atre, T. Han, S. Pascoli and B. Zhang, The search for heavy majorana neutrinos, JHEP 05 (2009) 030 [arXiv: 0901.3589] [INSPIRE].

[77] MEG collaboration, J. Adam et al., New constraint on the existence of the $\mu^{+} \rightarrow e^{+} \gamma$ decay, Phys. Rev. Lett. 110 (2013) 201801 [arXiv:1303.0754] [INSPIRE].

[78] EXO collaboration, M. Auger et al., Search for neutrinoless double-beta decay in ${ }^{136}$ Xe with EXO-200, Phys. Rev. Lett. 109 (2012) 032505 [arXiv: 1205.5608] [inSPIRE].

[79] EXO-200 collaboration, J.B. Albert et al., Search for Majorana neutrinos with the first two years of EXO-200 data, Nature $\mathbf{5 1 0}$ (2014) 229 [arXiv:1402.6956] [INSPIRE].

[80] KamLAND-Zen collaboration, A. Gando et al., Limit on neutrinoless $\beta \beta$ decay of Xe-136 from the first phase of KamLAND-Zen and comparison with the positive claim in Ge-76, Phys. Rev. Lett. 110 (2013) 062502 [arXiv:1211.3863] [INSPIRE].

[81] EXO collaboration, D. Tosi, The search for neutrino-less double-beta decay: summary of current experiments, arXiv:1402.1170 [INSPIRE].

[82] CUORE collaboration, P. Gorla, The CUORE experiment: status and prospects, J. Phys. Conf. Ser. 375 (2012) 042013 [INSPIRE].

[83] CUORE collaboration, D.R. Artusa et al., Searching for neutrinoless double-beta decay of ${ }^{130}$ Te with CUORE, arXiv:1402.6072 [INSPIRE].

[84] SNO+ collaboration, J. Hartnell, Neutrinoless double beta decay with SNO+, J. Phys. Conf. Ser. 375 (2012) 042015 [arXiv: 1201.6169] [INSPIRE].

[85] SuperNEMO collaboration, A. Barabash, SuperNEMO double beta decay experiment, J. Phys. Conf. Ser. 375 (2012) 042012 [inSPIRE].

[86] NEXT collaboration, F. Granena et al., NEXT, a HPGXe TPC for neutrinoless double beta decay searches, arXiv:0907.4054 [INSPIRE].

[87] NEXT collaboration, J.J. Gomez-Cadenas et al., Present status and future perspectives of the NEXT experiment, arXiv:1307.3914 [INSPIRE].

[88] J.F. Wilkerson et al., The Majorana demonstrator: a search for neutrinoless double-beta decay of germanium-76, J. Phys. Conf. Ser. 375 (2012) 042010 [INSPIRE]. 
[89] E. Bulbul et al., Detection of an unidentified emission line in the stacked X-ray spectrum of galaxy clusters, Astrophys. J. 789 (2014) 13 [arXiv: 1402.2301] [INSPIRE].

[90] A. Boyarsky, O. Ruchayskiy, D. Iakubovskyi and J. Franse, An unidentified line in X-ray spectra of the Andromeda galaxy and Perseus galaxy cluster, arXiv:1402.4119 [INSPIRE].

[91] G. Gelmini, E. Osoba, S. Palomares-Ruiz and S. Pascoli, MeV sterile neutrinos in low reheating temperature cosmological scenarios, JCAP 10 (2008) 029 [arXiv:0803.2735] [INSPIRE].

[92] B. Dasgupta and J. Kopp, Cosmologically safe eV-scale sterile neutrinos and improved dark matter structure, Phys. Rev. Lett. 112 (2014) 031803 [arXiv:1310.6337] [INSPIRE].

[93] M. Blennow, E. Fernandez-Martinez, J. Lopez-Pavon and J. Menendez, Neutrinoless double beta decay in seesaw models, JHEP 07 (2010) 096 [arXiv: 1005.3240] [INSPIRE].

[94] A. Abada and M. Lucente, Looking for the minimal inverse seesaw realisation, Nucl. Phys. B 885 (2014) 651 [arXiv:1401.1507] [INSPIRE].

[95] M.C. Gonzalez-Garcia and J.W.F. Valle, Fast decaying neutrinos and observable flavor violation in a new class of majoron models, Phys. Lett. B 216 (1989) 360 [INSPIRE].

[96] J.A. Casas and A. Ibarra, Oscillating neutrinos and muon $\rightarrow e, \gamma$, Nucl. Phys. B 618 (2001) 171 [hep-ph/0103065] [INSPIRE].

[97] D.V. Forero, S. Morisi, M. Tortola and J.W.F. Valle, Lepton flavor violation and non-unitary lepton mixing in low-scale type-I seesaw, JHEP 09 (2011) 142 [arXiv:1107.6009] [INSPIRE].

[98] M. Malinsky, T. Ohlsson and H. Zhang, Non-unitarity effects in a realistic low-scale seesaw model, Phys. Rev. D 79 (2009) 073009 [arXiv:0903.1961] [InSPIRE].

[99] P.S.B. Dev and R.N. Mohapatra, TeV scale inverse seesaw in $\mathrm{SO}(10)$ and leptonic non-unitarity effects, Phys. Rev. D 81 (2010) 013001 [arXiv:0910.3924] [INSPIRE].

[100] J. Kopp, P.A.N. Machado, M. Maltoni and T. Schwetz, Sterile neutrino oscillations: the global picture, JHEP 05 (2013) 050 [arXiv: 1303.3011] [INSPIRE].

[101] A. Abada, G. Arcadi and M. Lucente, Dark matter in the minimal inverse seesaw mechanism, arXiv:1406.6556 [INSPIRE]. 